\title{
BUSEMANN FUNCTIONS ON THE WASSERSTEIN SPACE
}

\author{
GUOMIN ZHU, WEN-LONG LI, AND XIAOJUN CUI
}

\begin{abstract}
We study rays and co-rays in the Wasserstein space $P_{p}(\mathcal{X})(p>1)$ whose ambient space $\mathcal{X}$ is a complete, separable, non-compact, locally compact length space. We show that rays in the Wasserstein space can be represented as probability measures concentrated on the set of rays in the ambient space. We show the existence of co-rays for any prescribed initial probability measure. We introduce Busemann functions on the Wasserstein space and show that co-rays are negative gradient lines in some sense.
\end{abstract}

\section{INTRODUCTION}

The Wasserstein distance plays an important role in optimal transport theory 3. 25, 29 and shows its advantages in numerous topics such as mean-field games 13, machine learning [4 and Hamilton-Jacobi equations 2, 19. Given an ambient space $\mathcal{X}$ which is a Polish space, namely complete separable metric space, the Wasserstein space $P_{p}(\mathcal{X})$ consisting of Borel probability measures on $\mathcal{X}$ with finite $p$-moment is also Polish [29, Theorem 6.18]. Besides, if $\mathcal{X}$ is a length (resp. geodesic) space, then $P_{p}(\mathcal{X})$ is also a length (resp. geodesic) space [22]. Lisini [22] also characterized geodesics in the Wasserstein space as Borel probability measures on $C([0, T] ; \mathcal{X})$ concentrated on the set of geodesics in $\mathcal{X}$. In the setting of $\mathcal{X}$ to be an Hadamard space, Bertrand and Kloeckner [9] investigated the geometry of $P_{2}(\mathcal{X})$, especially rays and geodesic boundary.

Busemann functions, introduced by Busemann [12, are powerful tools for studying the topology and geometry of some kinds of non-compact spaces. For example, Cheeger and Gromoll 14 used them to prove the celebrated splitting theorem for manifolds of nonnegative Ricci curvature. Bangert [5] investigated the dynamics on a Riemannian 2-torus by means of Busemann functions on the covering space. For more applications of Busemann functions, we refer to [6, 7, 20, 24, 26, 27, 28. Furthermore the Busemann function has been introduced into the study of Lorentzian geometry $[8,18,21$. Also, on a non-compact complete Riemannian manifold, they are viscosity solutions to the Eikonal equation (see [16]).

The aim of this paper is to extend Busemann functions to the Wasserstein space and thereby to get similar results as in the conventional case. In a metric space $(\mathcal{Y}, d)$, a ray is a curve $\gamma \in C\left(\mathbb{R}_{+} ; \mathcal{Y}\right)$ satisfying $d\left(\gamma_{s}, \gamma_{t}\right)=|s-t| d\left(\gamma_{0}, \gamma_{1}\right)$ for any $s, t \geq 0$ where $k_{\gamma}:=d\left(\gamma_{0}, \gamma_{1}\right)$ is called the speed of $\gamma$. Another ray $\tilde{\gamma}$ is said to be

Date: October 15, 2019.

2010 Mathematics Subject Classification. 58E10, 60B10, 60H30.

Key words and phrases. Co-ray, Wasserstein space, Busemann function.

The third author is supported by the National Natural Science Foundation of China (Grants 11571166, 11631006, 11790272). The Project Funded by the Priority Academic Program Development of Jiangsu Higher Education Institutions (PAPD) and the Fundamental Research Funds for the Central Universities. 
a co-ray from $p$ to $\gamma$ if it is the limit of a sequence $\left\{\zeta^{n}\right\}$ as $n \rightarrow \infty$, where $\zeta^{n}$ is a geodesic connecting $p^{n}$ and $\gamma_{t_{n}}$ with $p^{n} \rightarrow p$ and $t_{n} \rightarrow+\infty$. Usually a Busemann function is determined by a ray. As a preparation we give a characterization of rays in the Wasserstein space, that is, every ray in $P_{p}(\mathcal{X})$ can be represented by a random ray in $\mathcal{X}$. The main ingredient in the study of Busemann functions is co-ray. A common method to show the existence of co-rays is selecting a sequence of geodesics with some good properties which by Ascoli's theorem admits a subsequence converging to a co-ray. In the case of $P_{p}(\mathcal{X})$, the essential difficulty lies in the absence of local compactness: any non-compact Wasserstein space can not be locally compact [3, Remark 7.1.9]. Meanwhile this fact implies that a Wasserstein space is not a G-space on which the Busemann function is initially defined, because G-spaces are finitely compact i.e., every bounded infinity set has at least one accumulation point.

Without local compactness, our approach consists of two steps. First we construct probability measures $\Pi^{n}$ on $C\left(\mathbb{R}_{+} ; \mathcal{X}\right)$ representing the geodesics $\zeta^{n}$. The sequence $\left\{\Pi^{n}\right\}$ admits some accumulation points in the sense of weak convergence if it is confirmed to be tight. Then we show that time sections of the weak convergent subsequences are actually uniformly integrable, which means that the associated geodesics are convergent pointwisely with respect to the Wasserstein distance $W_{p}$.

With the assumptions of $p>1$ and $\mathcal{X}$ to be a complete, separable, non-compact, locally compact length space, main results of this paper are stated as follows.

Theorem 1.1. Each point in $P_{p}(\mathcal{X})$ is the origin of at least one unit-speed ray.

$\mathcal{X}$ is called non-branching if any geodesic $\zeta:[a, b] \rightarrow \mathcal{X}$ is uniquely determined by its restriction to a nontrivial time-interval.

Theorem 1.2. Let $\left(\mu_{t}\right)_{t \geq 0}$ be a unit-speed ray in $P_{p}(\mathcal{X})$.

(i) For any $\nu_{0} \in P_{p}(\mathcal{X})$, there exists at least one co-ray from $\nu_{0}$ to $\left(\mu_{t}\right)_{t \geq 0}$;

(ii) Moreover if $\mathcal{X}$ is non-branching, let $\left(\nu_{t}\right)_{t \geq 0}$ be one of the co-rays, then for any $\tau>0$ the subray $\left(\nu_{t+\tau}\right)_{t \geq 0}$ is the unique co-ray from $\nu_{\tau}$ to $\left(\mu_{t}\right)_{t \geq 0}$.

For a unit-speed ray $\left(\mu_{t}\right)_{t \geq 0}$ in $P_{p}(\mathcal{X})$, the Busemann function

$$
b_{\mu}(\nu)=\lim _{t \rightarrow+\infty}\left[W_{p}\left(\nu, \mu_{t}\right)-t\right]
$$

is well defined on $P_{p}(\mathcal{X})$.

Theorem 1.3. Let $\left(\mu_{t}\right)_{t \geq 0}$ and $\left(\nu_{t}\right)_{t \geq 0}$ be two unit-speed rays in $P_{p}(\mathcal{X})$. Assume $\mathcal{X}$ is non-branching, then $\left(\nu_{t}\right)_{t \geq 0}$ is a co-ray from $\nu_{0}$ to $\left(\mu_{t}\right)_{t \geq 0}$ if and only if

$$
b_{\mu}\left(\nu_{t}\right)-b_{\mu}\left(\nu_{s}\right)=s-t
$$

holds for any $s, t \geq 0$.

This paper is organized as follows. Section 2 presents some preliminaries. In Section 3 we study the structure of rays in the Wasserstein space. More precisely, we show that each ray can be represented as a probability measure concentrated on the set of rays in the ambient space $\mathcal{X}$. Section 4 presents the existence of co-rays in $P_{p}(\mathcal{X})$ and thus Theorem 1.1 and Theorem 1.2 (i) are proved. To prove a sequence of geodesic is compact in the pointwise convergence topology with respect to $W_{p}$, the key point is to show the uniformly integrability of the projections at any fixed time $\tau \geq 0$. In Section 5 we define the Busemann function on the Wasserstein space and obtain some analogue fundamental properties to the conventional case. The proofs of Theorem 1.2 (ii) and Theorem 1.3 are completed in this section. 


\section{Preliminaries}

2.1. Convergence in the Wasserstein space. Let $(\mathcal{X}, d)$ be a Polish space. For $p \geq 1$, the Wasserstein space $P_{p}(\mathcal{X})$ of order $p$ is the set of Borel probability measures with finite $p$-moments, i.e.,

$$
P_{p}(\mathcal{X})=\left\{\mu \in P(\mathcal{X}) \mid \int_{\mathcal{X}} d\left(x_{0}, x\right)^{p} d \mu(x)<+\infty\right\},
$$

where $x_{0} \in \mathcal{X}$ is fixed. This space does not depend on the choice of $x_{0}$. Given $\mu$, $\nu \in P_{p}(\mathcal{X})$, we denote by $\Pi(\mu, \nu)$ the set of Borel probability measure on $\mathcal{X} \times \mathcal{X}$ whose marginals are $\mu$ and $\nu$ respectively. Elements in $\Pi(\mu, \nu)$ are called couplings of $(\mu, \nu)$. The Wasserstein distance of order $p$ between $\mu$ and $\nu$ is defined by

$$
W_{p}(\mu, \nu)=\left(\min _{\pi \in \Pi(\mu, \nu)} \int_{\mathcal{X}} d(x, y)^{p} d \pi(x, y)\right)^{1 / p} .
$$

A coupling $\pi$ is said to be optimal if it achieves the minimum.

The space $C([0, T] ; \mathcal{X})$ of continuous curves in $\mathcal{X}$ equipped with the metric $\rho_{T}$ is a Polish space, where

$$
\rho_{T}(\alpha, \beta)=\sup _{0 \leq t \leq T} d(\alpha(t), \beta(t)), \text { for } \alpha, \beta \in C([0, T] ; \mathcal{X}) .
$$

For $\alpha, \beta \in C\left(\mathbb{R}_{+} ; \mathcal{X}\right)$, we define

$$
\rho(\alpha, \beta)=\sum_{N \in \mathbb{N}} 2^{-N} \frac{\rho_{N}(\alpha, \beta)}{1+\rho_{N}(\alpha, \beta)} .
$$

Then $\left(C\left(\mathbb{R}_{+} ; \mathcal{X}\right), \rho\right)$ is also a Polish space 30 .

Let $P(\mathcal{X})$ denote the set of Borel probability measures on $\mathcal{X}$. The support of $\mu \in P(\mathcal{X})$ defined by

$$
\operatorname{supp} \mu=\left\{x \in \mathcal{X} \mid \mu\left(B_{r}(x)\right)>0 \text { for } r>0\right\}
$$

is the smallest closed set on which $\mu$ is concentrated. We say that a sequence $\left\{\mu^{n}\right\} \subset P(\mathcal{X})$ converges weakly to $\mu \in P(\mathcal{X})$, denoted by $\mu^{n} \Rightarrow \mu$, if

$$
\lim _{n \rightarrow \infty} \int_{\mathcal{X}} f(x) d \mu_{n}(x)=\int_{\mathcal{X}} f(x) d \mu(x)
$$

for every bounded continuous function $f$ on $\mathcal{X}$.

Proposition 2.1 (3, Proposition 5.1.8]). If $\left\{\mu^{n}\right\} \subset P(\mathcal{X})$ converges weakly to $\mu \in P(\mathcal{X})$, then for any $x \in \operatorname{supp} \mu$ there exists a sequence $x^{n} \in \operatorname{supp} \mu^{n}$ such that $\lim _{n \rightarrow \infty} x^{n}=x$.

$S \subset P(\mathcal{X})$ is said to be tight if for any $\varepsilon>0$, there exists a compact set $K_{\varepsilon} \subset \mathcal{X}$ such that $\mu\left[K_{\varepsilon}\right]>1-\varepsilon$ for every $\mu \in P(\mathcal{X})$. Every single probability measure on a Polish space is itself tight (see e.g. [10, Theorem 1.3]). The following theorem indicates why the tightness makes sense.

Theorem 2.2 (Prokhorov). Let $\mathcal{X}$ be a Polish space. $S \subset P(\mathcal{X})$ is tight if and only if it is relatively compact in $P(\mathcal{X})$.

There is a tightness criterion for a sequence of probability measures on the space $C\left(\mathbb{R}_{+} ; \mathcal{X}\right)$. 
Theorem 2.3 ([30, Theorem 4]). Let $\left\{P^{n}\right\}$ be a sequence of probability measures on $C\left(\mathbb{R}_{+} ; \mathcal{X}\right)$. Then $\left\{P^{n}\right\}$ is tight if and only if these two conditions hold:

(i) For each $t \geq 0$ and $\eta>0$, there exists a compact set $K_{t}$ in $\mathcal{X}$ such that

$$
P^{n}\left\{x \in C\left(\mathbb{R}_{+} ; \mathcal{X}\right) \mid x(t) \in K_{t}\right\}>1-\eta, \text { for any } n \geq 1 .
$$

(ii) For each $j \geq 1$ and $\varepsilon, \eta>0$, there exists a $\delta \in(0,1)$, and an $n_{0} \in \mathbb{N}$ such that

$$
P^{n}\left\{x \in C\left(\mathbb{R}_{+} ; \mathcal{X}\right) \mid w_{x}^{j}(\delta) \geq \varepsilon\right\} \leq \eta, \text { for any } n \geq n_{0},
$$

where $w_{x}^{j}(\delta)=\sup _{\substack{0 \leq s, t \leq j \\|s-t|<\delta}} d(x(s), x(t))$.

The next theorem provides a more effective description of the convergence with respect to the Wasserstein distance.

Theorem 2.4 ([3, Theorem 7.1.5]). Let $(\mathcal{X}, d)$ be a Polish space and $p \geq 1$. Let $\left\{\mu^{n}\right\}_{n \in \mathbb{N}} \subset P_{p}(\mathcal{X})$ and $\mu \in P_{p}(\mathcal{X})$, then the following statements are equivalent:

(i) for some $x_{0} \in \mathcal{X}, \mu^{n} \Rightarrow \mu$ and

$$
\lim _{R \rightarrow \infty} \limsup _{n \rightarrow \infty} \int_{d\left(x_{0}, x\right) \geq R} d\left(x_{0}, x\right)^{p} d \mu^{n}(x)=0 ;
$$

(ii) $W_{p}\left(\mu^{n}, \mu\right) \rightarrow 0$ as $n \rightarrow \infty$.

2.2. Geodesics in the Wasserstein space. Let $(\mathcal{Y}, d)$ be a metric space. The length of a continuous curve $\zeta:[a, b] \rightarrow \mathcal{Y}$ is defined by

$$
L(\zeta)=\sup _{N \in \mathbb{N} a=t_{0}<t_{1}<\cdots<t_{N}=b} \sum_{i=0}^{N-1} d\left(\zeta_{t_{i}}, \zeta_{t_{i+1}}\right) .
$$

$(\mathcal{Y}, d)$ is said to be a length space if for any $x, y \in \mathcal{Y}$,

$$
d(x, y)=\inf _{\zeta \in C([0,1] ; \mathcal{Y})}\left\{L(\zeta) \mid \zeta_{0}=x, \zeta_{1}=y\right\} .
$$

$\mathcal{Y}$ is a geodesic space if the infimum in equation (2.5) is attainable for any $x, y \in \mathcal{Y}$. $\zeta$ is called a constant-speed minimizing geodesic segment if

$$
d\left(\zeta_{s}, \zeta_{t}\right)=\frac{|t-s|}{b-a} d\left(\zeta_{a}, \zeta_{b}\right) \text { for any } s, t \in[a, b] .
$$

For convenience, throughout this paper we use the single word "geodesic" instead.

The next statement is a straight corollary to [29, Corollary 7.22] via simple reparameterization. This conclusion is twofold: The Wasserstein space over a complete separable locally compact length space is a geodesic space; Geodesics in such a Wasserstein space can be considered as probability measures concentrated on the set of geodesics in the ambient space.

Proposition 2.5. Let $p>1$ and let $(\mathcal{X}, d)$ be a complete separable, locally compact length space. Given $\mu, \nu \in P_{p}(\mathcal{X})$, let $L=W_{p}(\mu, \nu)$. Then for any continuous curve $\left(\mu_{t}\right)_{0 \leq t \leq L}$ in $P(\mathcal{X})$ with $\mu_{0}=\mu, \mu_{L}=\nu$, the following properties are equivalent:

(i) $\mu_{t}$ is the law of $\zeta_{t}$, where $\zeta:[0, L] \rightarrow \mathcal{X}$ is a random geodesic such that $\left(\zeta_{0}, \zeta_{L}\right)$ is an optimal coupling;

(ii) $\left(\mu_{t}\right)_{0 \leq t \leq L}$ is a unit-speed geodesic in the space $P_{p}(\mathcal{X})$.

Moreover, for any given $\mu, \nu \in P_{p}(\mathcal{X})$, there exists at least one such curve. We denote by $T(\mu, \nu)$ the set of unit-speed geodesics from $\mu$ to $\nu$. 
Let $f: \mathcal{Y}_{1} \rightarrow \mathcal{Y}_{2}$ be a Borel map between Polish spaces, and $\lambda$ be a Borel measure on $\mathcal{Y}_{1}$. The push-forward of $\lambda$, denoted by $f_{\#} \lambda$, is defined by $\left(f_{\#} \lambda\right)[A]=\lambda\left[f^{-1}(A)\right]$ for any Borel subset $A$. In the case of Proposition 2.5. let $\Pi$ be the law of $\zeta$, then $\mu_{t}=\left(e_{t}\right)_{\#} \Pi$ where $e_{t}: \zeta \mapsto \zeta_{t}$ be the canonical projection. Besides, this work naturally prompts us to study rays in the Wasserstein space.

\section{Characterization of Rays in the Wasserstein space}

In the rest of this paper, we always assume that $(\mathcal{X}, d)$ is a complete, separable non-compact, locally compact length space and the order $p>1$. Recall that a ray in $\mathcal{X}$ is a curve $\gamma \in C\left(\mathbb{R}_{+} ; \mathcal{X}\right)$ satisfying

$$
d\left(\gamma_{s}, \gamma_{t}\right)=|t-s| d\left(\gamma_{0}, \gamma_{1}\right) \text { for any } s, t \geq 0,
$$

where $k_{\gamma}=d\left(\gamma_{0}, \gamma_{1}\right)$ is called the speed of $\gamma$.

Lemma 3.1. Let $\Gamma$ be the set of rays in $\mathcal{X}$, then $\Gamma$ is closed in $\left(C\left(\mathbb{R}_{+} ; \mathcal{X}\right), \rho\right)$. As a consequence, $(\Gamma, \rho)$ is a Polish space.

Proof. Let $\left\{\gamma^{n}\right\}$ be a Cauchy sequence of $\Gamma$, then there exists a $\gamma: \mathbb{R}_{+} \rightarrow \mathcal{X}$ such that $\rho\left(\gamma^{n}, \gamma\right) \rightarrow 0$. Denote $k=\lim _{n \rightarrow \infty} d\left(\gamma_{0}^{n}, \gamma_{1}^{n}\right)$. The limit exists since $\lim _{n \rightarrow \infty} d\left(\gamma_{t}^{n}, \gamma_{t}\right)=0$ for any $t \geq 0$. For any $t_{1}, t_{2} \geq 0$,

$$
\left|d\left(\gamma_{t_{1}}^{n}, \gamma_{t_{2}}^{n}\right)-k\right| t_{1}-t_{2}||=\left|t_{1}-t_{2}\right|\left|d\left(\gamma_{0}^{n}, \gamma_{1}^{n}\right)-k\right| \rightarrow 0 .
$$

By the triangle inequality,

$$
\begin{aligned}
\left|d\left(\gamma_{t_{1}}, \gamma_{t_{2}}\right)-d\left(\gamma_{t_{1}}^{n}, \gamma_{t_{2}}^{n}\right)\right| & =\left|d\left(\gamma_{t_{1}}, \gamma_{t_{2}}\right)-d\left(\gamma_{t_{1}}^{n}, \gamma_{t_{2}}^{n}\right)\right| \\
& \leq d\left(\gamma_{t_{1}}^{n}, \gamma_{t_{1}}\right)+d\left(\gamma_{t_{2}}^{n}, \gamma_{t_{2}}\right) \rightarrow 0 .
\end{aligned}
$$

Combining (3.2) and (3.3), we have $\left|d\left(\gamma_{t_{1}}, \gamma_{t_{2}}\right)-k\right| t_{1}-t_{2}||=0$, then the conclusion follows.

As shown in Proposition 2.5, if geodesics in $P_{p}(\mathcal{X})$ do not share the same lengths, then their corresponding random curves are defined on different time intervals. So we introduce the mapping $E_{T}: C([0, T] ; \mathcal{X}) \rightarrow C\left(\mathbb{R}_{+} ; \mathcal{X}\right)$ by

$$
\left(E_{T}(\zeta)\right)_{s}=\zeta_{\min \{s, T\}}, s \geq 0
$$

in order to extend their sample paths onto the common space $C\left(\mathbb{R}_{+} ; \mathcal{X}\right)$.

Definition 3.2. Let $\left(\mu_{t}\right)_{0 \leq t \leq L}$ be a geodesic in $P_{p}(\mathcal{X})$ and $\pi$ be a probability measure on $C([0, L] ; \mathcal{X})$ such that $\mu_{t}=\left(e_{t}\right)_{\#} \pi$ for $0 \leq t \leq L$. The probability measure $\left(E_{L}\right)_{\#} \pi$ on $C\left(\mathbb{R}_{+} ; \mathcal{X}\right)$ is called a lifting of the geodesic.

It can be seen from Proposition 2.5 that each geodesic in $P_{p}(\mathcal{X})$ admits a lifting.

Lemma 3.3. For $\mu, \nu \in P_{p}(\mathcal{X})$, let $\pi$ be an optimal coupling. Then for $R>0$,

$$
\pi\{(x, y) \mid d(x, y)>R\} \leq\left[\frac{W_{p}(\mu, \nu)}{R}\right]^{p} .
$$

Proof. Given $R>0$, by the definition of $W_{p}$,

$$
\begin{aligned}
\int_{d(x, y)>R} R^{p} d \pi(x, y) & \leq \int_{d(x, y)>R} d(x, y)^{p} d \pi(x, y) \\
& \leq \int_{\mathcal{X} \times \mathcal{X}} d(x, y)^{p} d \pi(x, y) \\
& =W_{p}^{p}(\mu, \nu) .
\end{aligned}
$$


So we obtain the inequality (3.4).

Theorem 3.4. Let $\left\{\nu^{n}\right\}$ and $\left\{\mu^{n}\right\}$ be sequences in $P_{p}(\mathcal{X})$ such that $\left\{\nu^{n}\right\}$ is tight and $L_{n}:=W_{p}\left(\mu^{n}, \nu^{n}\right) \rightarrow+\infty$. For each $n$, let $\Pi^{n}$ be a lifting of an element in $T\left(\nu^{n}, \mu^{n}\right)$, then $\left\{\Pi^{n}\right\}$ is tight.

Proof. Let $\nu_{t}^{n}=\left(e_{t}\right)_{\#} \Pi^{n}$ for $t \leq L_{n}$. Fix an arbitrary $t>0$.

(i) Given any $\eta>0$, there exists an $N$ such that $L_{n} \geq t$ for $n>N$. Recall that each single probability measure on a Polish space is tight. For $n \leq N$, there exists a compact set $K_{t}^{n}$ such that

$$
\Pi^{n}\left\{\gamma^{n} \in C\left(\mathbb{R}_{+} ; \mathcal{X}\right) \mid \gamma_{t}^{n} \in K_{t}^{n}\right\}=\nu_{t}^{n}\left[K_{t}^{n}\right]>1-\frac{\eta}{2 N} .
$$

While for $n>N$, by the tightness of $\left\{\nu_{0}^{n}\right\}$ there exists a constant $D_{0}$ such that

$$
\Pi^{n}\left\{\gamma^{n} \mid d\left(\gamma_{0}^{n}, x_{0}\right)>D_{0}\right\}=\nu_{0}^{n}\left\{x \mid d\left(x, x_{0}\right)>D_{0}\right\}<\frac{\eta}{4} .
$$

By Proposition 2.5, for each $n \in \mathbb{N}, \pi^{n}:=\left(e_{0}, e_{L_{n}}\right)_{\#} \Pi^{n}$ is an optimal coupling of $\left(\nu^{n}, \mu^{n}\right)$. By Lemma 3.3.

$$
\Pi^{n}\left\{\gamma^{n} \mid d\left(\gamma_{0}^{n}, \gamma_{t}^{n}\right)>R\right\}=\pi^{n}\{(x, y) \mid d(x, y)>R\} \leq\left(\frac{t}{R}\right)^{p} .
$$

For $D_{t} \geq t(4 / \eta)^{1 / p}+D_{0}$, from (3.6) and (3.7) we have

$$
\begin{aligned}
& \Pi^{n}\left\{\gamma^{n} \mid d\left(\gamma_{t}^{n}, x_{0}\right)>D_{t}\right\} \\
\leq & \Pi^{n}\left\{\gamma^{n} \mid d\left(\gamma_{0}^{n}, x_{0}\right)>D_{0}\right\}+\Pi^{n}\left\{\gamma^{n} \mid d\left(\gamma_{0}^{n}, x_{0}\right) \leq D_{0}, d\left(\gamma_{0}^{n}, \gamma_{t}^{n}\right)>D_{t}-D_{0}\right\} \\
\leq & \Pi^{n}\left\{\gamma^{n} \mid d\left(\gamma_{0}^{n}, x_{0}\right)>D_{0}\right\}+\Pi^{n}\left\{\gamma^{n} \mid d\left(\gamma_{0}^{n}, \gamma_{t}^{n}\right)>D_{t}-D_{0}\right\} \\
\leq & \frac{\eta}{4}+\left(\frac{t}{D_{t}-D_{0}}\right)^{p} \\
\leq & \eta / 2
\end{aligned}
$$

which means there is a compact set $K_{t}=\left\{x \mid d\left(x, x_{0}\right) \leq D_{t}\right\} \cup \bigcap_{i=1}^{N} K_{t}^{i}$ such that

$$
\Pi^{n}\left\{\gamma^{n} \mid \gamma^{n} \in K_{t}\right\}>1-\eta .
$$

(ii) For any fixed $j \geq 1$, by Definition [3.2, for $n>j$ the curves $\left(\nu_{t}^{n}\right)_{0 \leq t \leq j}$ are geodesics in $P_{p}(\mathcal{X})$. For any fixed $\varepsilon, \eta>0$, let $\delta<\varepsilon \eta^{1 / p}$, then

$$
\Pi^{n}\left\{\gamma^{n} \mid w_{\gamma^{n}}^{j}(\delta)>\varepsilon\right\}=\Pi^{n}\left\{\gamma^{n} \mid \sup _{\substack{0 \leq s, t \leq j \\|s-t|<\delta}} d\left(\gamma_{s}^{n}, \gamma_{t}^{n}\right)>\varepsilon\right\} \leq\left(\frac{\delta}{\varepsilon}\right)^{p}<\eta
$$

by using Lemma 3.3 again.

Hence the tightness of $\left\{\Pi^{n}\right\}$ follows from Theorem 2.3

Now we are able to characterize rays in $P_{p}(\mathcal{X})$. A similar representation can be found in [9, Proposition 3.2] for the 2-Wasserstein space over an Hadamard space.

Corollary 3.5. The following statements are equivalent:

(i) $\left(\mu_{t}\right)_{t \geq 0}$ is a ray in $P_{p}(\mathcal{X})$;

(ii) For any $t_{1}, t_{2}$ with $0 \leq t_{1}<t_{2},\left(\mu_{t}\right)_{t_{1} \leq t \leq t_{2}}$ is a geodesic;

(iii) $\mu_{t}$ is the law of $\gamma_{t}$, where $\gamma$ is a random ray such that $\left(\gamma_{t_{1}}, \gamma_{t_{2}}\right)$ is an optimal coupling for any $t_{1}, t_{2}$ with $0 \leq t_{1}<t_{2}$. 
Proof. (i) and (ii) are equivalent by the definition of ray and (iii) $\Rightarrow$ (ii) is obvious.

(ii) $\Rightarrow$ (iii). For $j \in \mathbb{N}$, let $\Pi^{j}$ be a lifting of $\left(\mu_{t}\right)_{0 \leq t \leq j}$ and

$$
\Gamma^{j}=\{\zeta \in C([0, j] ; \mathcal{X}) \mid \zeta \text { is a geodesic }\} .
$$

By Proposition 2.5, $\Pi^{j}$ is concentrated on $E_{j}\left(\Gamma^{j}\right)$ such that $\left(e_{0}, e_{j}\right)_{\#} \Pi^{j}$ is an optimal coupling. By Theorem 3.4 and Theorem[2.2, it admits a subsequence $\left\{\Pi^{j^{\prime}}\right\}$ which converges weakly to some measure $\Pi$ on $C\left(\mathbb{R}_{+} ; \mathcal{X}\right)$. For any $\gamma \in \operatorname{supp} \Pi$, by Proposition 2.1, there exists $\zeta^{j^{\prime}} \in E_{j^{\prime}}\left(\Gamma^{j^{\prime}}\right)$ with

$$
\lim _{j^{\prime} \rightarrow \infty} \rho\left(\zeta^{j^{\prime}}, \gamma\right)=0
$$

For any $0 \leq s_{1}<s_{2}$, choose $T \geq \max \left\{1, s_{2}\right\}$, then (3.10) implies

$$
d\left(\zeta_{s}^{j^{\prime}}, \gamma_{s}\right) \rightarrow 0 \text { for any } s \in[0, T] .
$$

Notice that $d\left(\zeta_{s_{1}}^{j^{\prime}}, \zeta_{s_{2}}^{j^{\prime}}\right)=\left(s_{2}-s_{1}\right) d\left(\zeta_{0}^{j^{\prime}}, \zeta_{1}^{j^{\prime}}\right)$ when $j^{\prime}>T$. (3.11) yields

$$
d\left(\gamma_{s_{1}}, \gamma_{s_{2}}\right)=\left(s_{2}-s_{1}\right) d\left(\gamma_{0}, \gamma_{1}\right) \text {. }
$$

It follows that $\gamma \in \Gamma$, and consequently $\Pi$ is the law of a random ray.

For $0 \leq t_{1}<t_{2}$, denote $\pi_{t_{1}, t_{2}}^{j^{\prime}}=\left(e_{t_{1}}, e_{t_{2}}\right)_{\#} \Pi^{j^{\prime}}, \pi_{t_{1}, t_{2}}=\left(e_{t_{1}}, e_{t_{2}}\right)_{\#} \Pi$, then $\pi_{t_{1}, t_{2}}^{j^{\prime}} \Rightarrow \pi_{t_{1}, t_{2}}$. By the lower semicontinuity of the map $\pi \mapsto \int d^{p} d \pi$,

$$
\begin{aligned}
W_{p}^{p}\left(\mu_{t_{1}}, \mu_{t_{2}}\right) & \leq \int_{\mathcal{X} \times \mathcal{X}} d^{p}(x, y) d \pi_{t_{1}, t_{2}} \\
& \leq \liminf _{j^{\prime} \rightarrow \infty} \int_{\mathcal{X} \times \mathcal{X}} d^{p}(x, y) d \pi_{t_{1}, t_{2}}^{j^{\prime}} \\
& =W_{p}^{p}\left(\mu_{t_{1}}, \mu_{t_{2}}\right),
\end{aligned}
$$

which means $\pi_{t_{1}, t_{2}}$ is an optimal coupling.

\section{EXISTENCE OF CO-RAYS In The WASSERSTEIn SPACE}

In the conventional case, co-rays play a central role in the study of Busemann functions [12. This notion also make sense in the present case. The existence of co-rays in the Wasserstein space will be proved in this section.

Definition 4.1 (Co-ray). Let $\left(\mu_{t}\right)_{t \geq 0}$ be a unit-speed ray in $P_{p}(\mathcal{X})$. We say another ray $\left(\nu_{t}\right)_{t \geq 0}$ is a co-ray from $\nu_{0}$ to $\left(\mu_{t}\right)_{t \geq 0}$, if there exist:

- $\left\{t_{n}\right\} \subset \mathbb{R}_{+}$tends to infinity,

- $\left\{\nu_{0}^{n}\right\} \subset P_{p}(\mathcal{X})$ with $W_{p}\left(\nu_{0}^{n}, \nu_{0}\right) \rightarrow 0$,

- for $n \in \mathbb{N},\left(\nu_{t}^{n}\right)_{0 \leq t \leq L_{n}} \in T\left(\nu_{0}^{n}, \mu_{t_{n}}\right)$ where $L_{n}=W_{p}\left(\nu_{0}^{n}, \mu_{t_{n}}\right)$

such that $\lim _{n \rightarrow \infty} W_{p}\left(\nu_{t}^{n}, \nu_{t}\right)=0$ for every $t \geq 0$.

The gluing lemma (see e.g. 3, Lemma 5.3.2]) is often used in optimal transport to connect two couplings. However this instrument can not meet our demand while two random curves get involved, so we cite a theorem here in order to obtain another version of gluing lemma.

Theorem 4.2 (11, Theorem A.1]). Let $J$ be an arbitrary index set. For each $j \in J$, let $S_{j}, T_{j}$ be Polish spaces, $S=\prod_{j \in J} S_{j}, T=\prod_{j \in J} T_{j}$. Also, let $p_{j}: S \rightarrow S_{j}, q_{j}: T \rightarrow$ $T_{j}$ be canonical projections, $\phi_{j}: S_{j} \rightarrow T_{j}$ be a measurable map. If $\mu_{j}$ is a probability 
measure on $S_{j}$ and $\lambda$ is a probability measure on $T$ such that $\left(\phi_{j}\right)_{\#} \mu_{j}=\left(q_{j}\right)_{\#} \lambda$, $\forall j \in J$. Then there exists a probability measure $\sigma$ on $S$ such that:

(i) $p_{j} \sigma=\mu_{j}$, for all $j \in J$;

(ii) $\left(\left(\phi_{j} \circ p_{j}\right)_{j \in J}\right)_{\#} \sigma=\lambda$.

The modified gluing lemma allow us to glue a random curve and a coupling together.

Lemma 4.3. Let $\mathcal{Y}$ be a Polish space, $\alpha \in P(C([0, T] ; \mathcal{X})), \beta \in P(\mathcal{X} \times \mathcal{Y})$ and $\pi^{i}$ be the natural projection onto the $i$-th coordinate, $i=1,2$. If $\left(e_{T}\right)_{\#} \alpha=\left(\pi^{1}\right)_{\#} \beta$, then there exists a $\delta \in P(C([0, T] ; \mathcal{X}) \times \mathcal{Y})$ such that

$$
\left(\pi^{1}\right)_{\#} \delta=\alpha,\left(e_{T} \pi^{1}, \pi^{2}\right)_{\#} \delta=\beta .
$$

Proof. Let $S_{1}=C([0, T] ; \mathcal{X}), T_{1}=\mathcal{X}, \phi_{1}=e_{T} ; S_{2}=\mathcal{Y}=T_{2}, \phi_{2}=\operatorname{Id} \mathcal{Y} ; \mu_{1}=$ $\alpha, \mu_{2}=\left(\pi^{2}\right)_{\#} \beta=\left(q_{2}\right)_{\#} \beta, \lambda=\beta$. Then

$$
\begin{gathered}
\left(\phi_{1}\right)_{\#} \mu_{1}=\left(e_{T}\right)_{\#} \alpha=\left(\pi^{1}\right)_{\#} \beta=\left(q_{1}\right)_{\#} \beta ; \\
\left(\phi_{1}\right)_{\#} \mu_{2}=(\operatorname{Id} \mathcal{Y})_{\#}\left(\left(q_{2}\right)_{\#} \beta\right)=\left(q_{2}\right)_{\#} \beta .
\end{gathered}
$$

Applying Theorem 4.2 for $J=\{1,2\}$, there exists a probability measure $\delta$ on $S_{1} \times S_{2}=C([0, T] ; \mathcal{X}) \times \mathcal{Y}$ such that

$$
\begin{gathered}
\left(\pi^{1}\right)_{\#} \delta=\left(p_{1}\right)_{\#} \delta=\mu_{1}=\alpha, \\
\left(e_{T} \pi^{1}, \pi^{2}\right)_{\#} \delta=\left(\phi_{1} \circ p_{1}, \phi_{1} \circ p_{2}\right)_{\#}=\lambda=\beta .
\end{gathered}
$$

Theorem 4.4. Let $\left(\mu_{t}\right)_{t \geq 0}$ be a unit-speed ray in $P_{p}(\mathcal{X}), p>1$. Given an arbitrary $\nu_{0} \in P_{p}(\mathcal{X})$, for any:

- $\left\{t_{n}\right\}$ increasing to infinity,

- $\left\{\nu_{0}^{n}\right\} \subset P_{p}(\mathcal{X})$ with $W_{p}\left(\nu^{n}, \nu_{0}\right) \rightarrow 0$,

- $\left(\nu_{t}^{n}\right)_{0 \leq t \leq L_{n}} \in T\left(\nu_{0}^{n}, \mu_{t_{n}}\right), n \in \mathbb{N}$ where $L_{n}=W_{p}\left(\nu_{0}^{n}, \mu_{t_{n}}\right)$,

there exists a subsequence of $\left\{\left(\nu_{t}^{n}\right)_{0 \leq t \leq L_{n}}\right\}$ which converges to a co-ray from $\nu_{0}$ to $\left(\mu_{t}\right)_{t \geq 0}$.

Proof. Let $\Lambda^{n}$ be a lifting of $\left(\nu_{t}^{n}\right)_{0 \leq t \leq L_{n}}$. By Theorem 3.4. there exists a subsequence of $\left\{\Lambda^{n}\right\}$, still denoted by the same notation, which converges weakly to a probability measure $\Lambda$ on $C(\mathcal{X})$. Then for arbitrary fixed $\tau \geq 0$,

$$
\nu_{\tau}^{n}=\left(e_{\tau}\right)_{\#} \Lambda^{n} \Rightarrow\left(e_{\tau}\right)_{\#} \Lambda:=\nu_{\tau} .
$$

To show that $W_{p}\left(\nu_{\tau}^{n}, \nu_{\tau}\right) \rightarrow 0$ as $n \rightarrow \infty$, by Theorem 2.4 it remains to prove that

$$
\lim _{R \rightarrow \infty} \limsup _{n \rightarrow \infty} \int_{d\left(x_{0}, z\right) \geq R} d\left(x_{0}, z\right)^{p} d \nu_{\tau}^{n}(z)=0 .
$$

By Proposition 2.5, for each $n$, there exists $\alpha^{n} \in P\left(C\left(\left[0, L_{n}\right] ; \mathcal{X}\right)\right)$ satisfying

$$
\left(e_{t}\right)_{\#} \alpha^{n}=\nu_{t}^{n} \text { for } t \in\left[0, L_{n}\right] \text {. }
$$

By Corollary 3.5, there is a random ray $\left(\eta_{t}\right)_{t \geq 0}$ such that $\left(\eta_{0}, \eta_{t}\right)$ are optimal couplings and $\mu_{t}=$ law $\left(\eta_{t}\right)$ for any $t \geq 0$. We write $\beta$ for the distribution of $\eta$. For each $n$, let $\beta^{n}=\left(e_{0}, e_{t_{n}}\right)_{\#} \beta$, then it is an optimal coupling of $\left(\mu_{0}, \mu_{t_{n}}\right)$. By Lemma 4.3. we can construct a sequence of probability measures $\left\{\Pi^{n}\right\} \subset P\left(C\left(\left[0, L_{n}\right] ; \mathcal{X}\right) \times \mathcal{X}\right)$ satisfying

$$
\left(\pi^{1}\right)_{\#} \Pi^{n}=\alpha^{n},\left(e_{L_{n}} \pi^{1}, \pi^{2}\right)_{\#} \Pi^{n}=\beta^{n} .
$$


By the triangle inequality,

$$
\begin{aligned}
\left|L_{n}-t_{n}\right| & =\left|W_{p}\left(\nu_{0}^{n}, \mu_{t_{n}}\right)-W_{p}\left(\mu_{0}, \mu_{t_{n}}\right)\right| \\
& \leq W_{p}\left(\nu_{0}^{n}, \mu_{0}\right) \\
& \leq W_{p}\left(\nu_{0}^{n}, \nu_{0}\right)+W_{p}\left(\nu_{0}, \mu_{0}\right) .
\end{aligned}
$$

Then

$$
\lim _{n \rightarrow \infty} \frac{t_{n}}{L_{n}}=1
$$

There exists an $N$ such that $L_{n}>\tau$ for all $n>N$. In this case, for $\Pi^{n}$-a.e. $\left(\gamma^{n}, y\right) \in C\left(\left[0, L_{n}\right] ; \mathcal{X}\right) \times \mathcal{X}$, by the triangle inequality,

$$
\begin{aligned}
d\left(x_{0}, \gamma_{\tau}^{n}\right) & \leq d\left(x_{0}, \gamma_{0}^{n}\right)+d\left(\gamma_{0}^{n}, \gamma_{\tau}^{n}\right) \\
& =d\left(x_{0}, \gamma_{0}^{n}\right)+\tau / L_{n} d\left(\gamma_{0}^{n}, \gamma_{L_{n}}^{n}\right) \\
& \leq d\left(x_{0}, \gamma_{0}^{n}\right)+\tau / L_{n}\left[d\left(x_{0}, \gamma_{0}^{n}\right)+d\left(x_{0}, y\right)+d\left(y, \gamma_{L_{n}}^{n}\right)\right] \\
& =\left(1+\frac{\tau}{L_{n}}\right) d\left(x_{0}, \gamma_{0}^{n}\right)+\frac{\tau}{L_{n}} d\left(x_{0}, y\right)+\frac{\tau}{L_{n}} d\left(y, \gamma_{L_{n}}^{n}\right) .
\end{aligned}
$$

The first equality follows from that each $\gamma^{n}$ is a random geodesic.

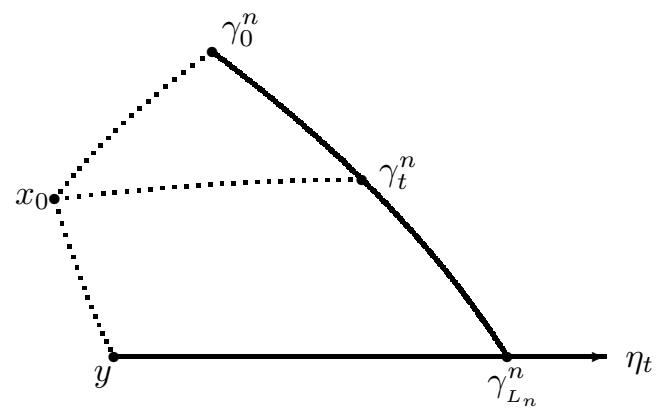

Applying Jensen's inequality we have

$$
\begin{aligned}
& \int_{d\left(x_{0}, z\right) \geq R} d^{p}\left(x_{0}, z\right) d \nu_{\tau}^{n}(z) \\
= & \int_{d\left(x_{0}, \gamma_{\tau}^{n}\right) \geq R} d^{p}\left(x_{0}, \gamma_{\tau}^{n}\right) d \Pi^{n}\left(\gamma^{n}, y\right) \\
\leq & 3^{p}\left(\frac{\tau}{L_{n}}+1\right)^{p} \int_{d\left(x_{0}, \gamma_{\tau}^{n}\right) \geq R} d^{p}\left(x_{0}, \gamma_{0}^{n}\right) d \Pi^{n}\left(\gamma^{n}, y\right) \\
& +\left(\frac{3 \tau}{L_{n}}\right)^{p} \int_{d\left(x_{0}, \gamma_{\tau}^{n}\right) \geq R} d^{p}\left(x_{0}, y\right) d \Pi^{n}\left(\gamma^{n}, y\right) \\
& +\left(\frac{3 \tau}{L_{n}}\right)^{p} \int_{d\left(x_{0}, \gamma_{\tau}^{n}\right) \geq R} d^{p}\left(y, \gamma_{L_{n}}^{n}\right) d \Pi^{n}\left(\gamma^{n}, y\right) \\
\leq & 3^{p}\left(\frac{\tau}{L_{n}}+1\right)^{p} \int_{d\left(x_{0}, \gamma_{\tau}^{n}\right) \geq R} d^{p}\left(x_{0}, \gamma_{0}^{n}\right) d \Pi^{n}\left(\gamma^{n}, y\right) \\
& +\left(\frac{3 \tau}{L_{n}}\right)^{p} W_{p}^{p}\left(\delta_{x_{0}}, \mu_{0}\right)+\left(\frac{3 \tau}{L_{n}}\right)^{p} \int_{d\left(x_{0}, \gamma_{\tau}^{n}\right) \geq R} d^{p}\left(y, \gamma_{L_{n}}^{n}\right) d \Pi^{n}\left(\gamma^{n}, y\right) \\
:= & 3^{p}\left(1+\frac{\tau}{L_{n}}\right)^{p} I_{1}+\left(\frac{3 \tau}{L_{n}}\right)^{p} W_{p}^{p}\left(\delta_{x_{0}}, \mu_{0}\right)+\left(\frac{3 \tau}{L_{n}}\right)^{p} I_{2} .
\end{aligned}
$$


We will consider the three terms above respectively.

(i) Let $M=\sup _{n}\left\{\frac{\tau}{L_{n}}\right\}$. For arbitrary $\varepsilon>0$, since $W_{p}\left(\nu_{0}^{n}, \nu_{0}\right) \rightarrow 0$, by Theorem 2.4 there exists an $R_{1}$ such that

$$
\limsup _{n \rightarrow \infty} \int_{d\left(x_{0}, z\right) \geq R_{1}} d^{p}\left(x_{0}, z\right) d \nu_{0}^{n}(z)<\frac{\varepsilon}{4 \cdot 3^{p}(1+M)^{p}} .
$$

We can further find an $R_{2}>R_{1}$ such that when $R>R_{2}$,

$$
\left(\frac{R_{1} \tau}{R-R_{1}}\right)^{p}<\frac{\varepsilon}{4 \cdot 3^{p}(1+M)^{p}}
$$

In this case we estimate $I_{1}$ as following:

$$
\begin{aligned}
I_{1}= & \int_{d\left(x_{0}, \gamma_{\tau}^{n}\right) \geq R} d^{p}\left(x_{0}, \gamma_{0}^{n}\right) d \Pi^{n}\left(\gamma^{n}, y\right) \\
= & \int_{d\left(x_{0}, \gamma_{0}^{n}\right) \geq R_{1}, d\left(x_{0}, \gamma_{\tau}^{n}\right) \geq R} d^{p}\left(x_{0}, \gamma_{0}^{n}\right) d \Pi^{n}\left(\gamma^{n}, y\right) \\
& +\int_{d\left(x_{0}, \gamma_{0}^{n}\right)<R_{1}, d\left(x_{0}, \gamma_{\tau}^{n}\right) \geq R} d^{p}\left(x_{0}, \gamma_{0}^{n}\right) d \Pi^{n}\left(\gamma^{n}, y\right) \\
\leq & \int_{d\left(x_{0}, \gamma_{0}^{n}\right) \geq R_{1}} d^{p}\left(x_{0}, \gamma_{0}^{n}\right) d \Pi^{n}\left(\gamma^{n}, y\right)+R_{1}^{p} \cdot \alpha^{n}\left\{\gamma^{n} \mid d\left(\gamma_{0}^{n}, \gamma_{\tau}^{n}\right)>R-R_{1}\right\} \\
\leq & \int_{d\left(x_{0}, z\right) \geq R_{1}} d^{p}\left(x_{0}, z\right) d \nu_{0}^{n}(z)+\left(\frac{R_{1} \tau}{R-R_{1}}\right)^{p} .
\end{aligned}
$$

The last inequality is obtained from Lemma 3.3. Consequently,

$$
\limsup _{n \rightarrow \infty} 3^{p}\left(1+\frac{\tau}{L_{n}}\right)^{p} I_{1} \leq \limsup _{n \rightarrow \infty} 3^{p}\left(1+\frac{\tau}{L_{n}}\right)^{p} \frac{\varepsilon}{2 \cdot 3^{p}(1+M)^{p}}<\frac{\varepsilon}{2} .
$$

(ii) Since $W_{p}\left(\mu_{0}, \mu_{1}\right)=1$, there exists an $R_{3}$ such that

$$
\int_{d\left(\eta_{0}, \eta_{1}\right) \geq R_{3}} d^{p}\left(\eta_{0}, \eta_{1}\right) d \beta(\eta)<\frac{\varepsilon}{6 \cdot(3 \tau)^{p}} .
$$

From the construction of $\Pi^{n}$, we have

$$
\begin{aligned}
& \int_{d\left(y, \gamma_{L_{n}}^{n}\right) \geq t_{n} R_{3}} d^{p}\left(y, \gamma_{L_{n}}^{n}\right) d \Pi^{n}\left(\gamma^{n}, y\right) \\
= & \int_{d\left(\eta_{0}, \eta_{t_{n}}\right) \geq t_{n} R_{3}} d^{p}\left(\eta_{0}, \eta_{t_{n}}\right) d \beta^{n}\left(\eta_{0}, \eta_{t_{n}}\right) \\
= & t_{n}^{p} \int_{d\left(\eta_{0}, \eta_{1}\right) \geq R_{3}} d^{p}\left(\eta_{0}, \eta_{1}\right) d \beta(\eta) .
\end{aligned}
$$

Due to the tightness of $\left\{\nu_{0}^{n}\right\}$, there is an $R_{4}>R_{3}$ with

$$
\nu_{0}^{n}\left\{z \mid d\left(x_{0}, z\right) \geq R_{4}\right\}<\frac{\varepsilon}{6 \cdot\left(3 \tau R_{3}\right)^{p}} \text { for all } n>N .
$$

Applying the inequality (3.4) again, we can obtain another $R_{5}>R_{4}$ such that when $R>R_{5}$,

$$
\alpha^{n}\left\{\gamma^{n} \mid d\left(\gamma_{0}^{n}, \gamma_{\tau}^{n}\right)>R-R_{4}\right\}<\frac{\varepsilon}{6 \cdot\left(3 \tau R_{3}\right)^{p}} \text { for all } n>N
$$


In this case,

$$
\begin{aligned}
I_{2}= & \int_{d\left(x_{0}, \gamma_{\tau}^{n}\right) \geq R} d^{p}\left(y, \gamma_{L_{n}}^{n}\right) d \Pi^{n}\left(\gamma^{n}, y\right) \\
= & \int_{d\left(x_{0}, \gamma_{\tau}^{n}\right) \geq R, d\left(y, \gamma_{L_{n}}^{n}\right) \geq t_{n} R_{3}} d^{p}\left(y, \gamma_{L_{n}}^{n}\right) d \Pi^{n}\left(\gamma^{n}, y\right) \\
& +\int_{d\left(x_{0}, \gamma_{\tau}^{n}\right) \geq R, d\left(y, \gamma_{L_{n}}^{n}\right)<t_{n} R_{3}, d\left(x_{0}, \gamma_{0}^{n}\right) \geq R_{4}} d^{p}\left(y, \gamma_{L_{n}}^{n}\right) d \Pi^{n}\left(\gamma^{n}, y\right) \\
& +\int_{d\left(x_{0}, \gamma_{\tau}^{n}\right) \geq R, d\left(y, \gamma_{L_{n}}^{n}\right)<t_{n} R_{3}, d\left(x_{0}, \gamma_{0}^{n}\right)<R_{4}} d^{p}\left(y, \gamma_{L_{n}}^{n}\right) d \Pi^{n}\left(\gamma^{n}, y\right) \\
\leq & \int_{d\left(y, \gamma_{L_{n}}^{n}\right) \geq t_{n} R_{3}} d^{p}\left(y, \gamma_{L_{n}}^{n}\right) d \Pi^{n}\left(\gamma^{n}, y\right) \\
& +\left(t_{n} R_{3}\right)^{p} \Pi^{n}\left\{\left(\gamma^{n}, y\right) \mid d\left(x_{0}, \gamma_{0}^{n}\right) \geq R_{4}\right\} \\
& +\left(t_{n} R_{3}\right)^{p} \Pi^{n}\left\{\left(\gamma^{n}, y\right) \mid d\left(\gamma_{0}^{n}, \gamma_{\tau}^{n}\right)>R-R_{4}\right\} \\
= & t_{n}^{p} \int_{d\left(\eta_{0}, \eta_{1}\right) \geq R_{3}} d^{p}\left(\eta_{0}, \eta_{1}\right) d \beta(\eta) \\
& +\left(t_{n} R_{3}\right)^{p} \nu_{0}^{n}\left\{z \mid d\left(x_{0}, z\right) \geq R_{4}\right\} \\
& +\left(t_{n} R_{3}\right)^{p} \alpha^{n}\left\{\gamma^{n} \mid d\left(\gamma_{0}^{n}, \gamma_{\tau}^{n}\right)>R-R_{4}\right\} .
\end{aligned}
$$

Combining this inequality with (4.7), (4.8), (4.9) and (4.3), we have

$$
\limsup _{n \rightarrow \infty}\left(\frac{3 \tau}{L_{n}}\right)^{p} I_{2} \leq \frac{\varepsilon}{2} \text {. }
$$

(iii) Notice that $\lim _{n \rightarrow \infty}\left(\frac{3 \tau}{L_{n}}\right)^{p} W_{p}^{p}\left(\delta_{x_{0}}, \mu_{0}\right)=0$. We obtain

$$
\limsup _{n \rightarrow \infty} \int_{d\left(x_{0}, z\right) \geq R} d^{p}\left(x_{0}, z\right) d \nu_{\tau}^{n}(z)<\varepsilon \text { when } R>\max \left\{R_{2}, R_{5}\right\} .
$$

We conclude from (4.1) that $\lim _{n \rightarrow \infty} W_{p}\left(\nu_{t}^{n}, \nu_{t}\right)=0$ for arbitrary $t \geq 0$, thus $\nu_{t} \in$ $P_{p}(\mathcal{X})$. Moreover, for $t, s \geq 0$,

$$
W_{p}\left(\nu_{t}, \nu_{s}\right)=\lim _{n \rightarrow \infty} W_{p}\left(\nu_{t}^{n}, \nu_{s}^{n}\right)=|t-s|,
$$

which means $\left(\nu_{t}\right)_{t \geq 0}$ is also a unit-speed ray in $P_{p}(\mathcal{X})$. Therefore it is a co-ray from $\nu_{0}$ to $\left(\mu_{t}\right)_{t \geq 0}$.

The first part of Theorem 1.2 follows directly from Theorem 4.4

A complete and locally compact length space, by the Hopf-Rinow Theorem (see [11, Theorem 2.5.28]), is boundedly compact i.e., every closed metric ball is compact. Then each point in $\mathcal{X}$ is the origin of some unit-speed rays, which is due to [23, Proposition 10.1.1]. It is easily seen that the mapping $x \mapsto \delta_{x}$ is an isometric embedding from $\mathcal{X}$ to $P_{p}(\mathcal{X})$. Let $\gamma$ be a unit-speed ray in $\mathcal{X}$ and $\mu_{t}=\delta_{\gamma_{t}}$, then $\left(\mu_{t}\right)_{t \geq 0}$ is a unit-speed ray in $P_{p}(\mathcal{X})$ accordingly. Notice that for each $\nu_{0} \in P_{p}(\mathcal{X})$, co-rays from $\nu_{0}$ to $\left(\mu_{t}\right)_{t \geq 0}$ are some of the unit-speed rays with the origin $\nu_{0}$, thus Theorem 1.1 holds. 


\section{Busemann functions on the Wasserstein Space}

Definition 5.1. Let $\left(\mu_{t}\right)_{t \geq 0}$ be a unit-speed ray in $P_{p}(\mathcal{X})$. The Busemann function associated with $\left(\mu_{t}\right)_{t \geq 0}$ is defined by

$$
b_{\mu}(\nu)=\lim _{t \rightarrow+\infty}\left[W_{p}\left(\nu, \mu_{t}\right)-t\right] .
$$

Remark 5.2. To show that the limit in (5.1) exists and is finite, by triangle inequality,

$$
W_{p}\left(\nu, \mu_{0}\right) \geq W_{p}\left(\mu_{0}, \mu_{t}\right)-W_{p}\left(\nu, \mu_{t}\right)=t-W_{p}\left(\nu, \mu_{t}\right) .
$$

For $0 \leq t_{1} \leq t_{2}$

$$
\left[t_{2}-W_{p}\left(\nu, \mu_{t_{2}}\right)\right]-\left[t_{1}-W_{p}\left(\nu, \mu_{t_{1}}\right)\right]=W_{p}\left(\mu_{t_{2}}, \mu_{t_{1}}\right)+W_{p}\left(\nu, \mu_{t_{2}}\right)-W_{p}\left(\nu, \mu_{t_{1}}\right) \geq 0 .
$$

Thereby $t-W_{p}\left(\nu, \mu_{t}\right)$ is bounded and non-decreasing with respect to $t$.

Remark 5.3. $b_{\mu}$ is a Lipschitz function since

$$
\left|b_{\mu}\left(\nu^{2}\right)-b_{\mu}\left(\nu^{1}\right)\right|=\lim _{t \rightarrow \infty}\left|W_{p}\left(\nu^{2}, \mu_{t}\right)-W_{p}\left(\nu^{1}, \mu_{t}\right)\right| \leq W_{p}\left(\nu^{1}, \nu^{2}\right) .
$$

Busemann functions have the following fundamental properties. For the conventional case, we refer to [24].

Proposition 5.4. Let $\left(\mu_{t}\right)_{t \geq 0}$ be a unit-speed ray in $P_{p}(\mathcal{X})$. If $\left(\nu_{t}\right)_{t \geq 0}$ is a co-ray from $\nu_{0}$ to $\left(\mu_{t}\right)_{t \geq 0}$, then

(i) $b_{\mu}\left(\nu_{t_{1}}\right)-b_{\mu}\left(\nu_{t_{2}}\right)=t_{2}-t_{1}$, for any $t_{1}, t_{2} \geq 0$;

(ii) $b_{\mu}(\lambda) \leq b_{\nu}(\lambda)+b_{\mu}\left(\nu_{0}\right)$, for each $\lambda \in P_{p}(\mathcal{X})$.

Proof. (i) Assume the geodesic sequence $\left(\nu_{t}^{n}\right)_{0 \leq t \leq L_{n}} \in T\left(\nu_{0}^{n}, \mu_{t_{n}}\right)$ converges to $\left(\nu_{t}\right)_{t \geq 0}$ as $n \rightarrow \infty$. Let $0 \leq t_{1} \leq t_{2}$, for any $n>t_{2}$ we have $W_{p}\left(\nu_{0}^{n}, \mu_{t_{n}}\right)=$ $W_{p}\left(\nu_{0}^{n}, \nu_{t_{i}}^{n}\right)+W_{p}\left(\nu_{t_{i}}^{n}, \mu_{t_{n}}\right), i=1,2$. Then

$$
\begin{aligned}
& \left|W_{p}\left(\nu_{t_{1}}, \mu_{t_{n}}\right)-W_{p}\left(\nu_{t_{2}}, \mu_{t_{n}}\right)-\left(t_{2}-t_{1}\right)\right| \\
= & \left|W_{p}\left(\nu_{t_{1}}, \mu_{t_{n}}\right)-W_{p}\left(\nu_{t_{2}}, \mu_{t_{n}}\right)-\left[W_{p}\left(\nu_{t_{1}}^{n}, \mu_{t_{n}}\right)-W_{p}\left(\nu_{t_{2}}^{n}, \mu_{t_{n}}\right)\right]\right| \\
\leq & W_{p}\left(\nu_{t_{1}}^{n}, \nu_{t_{1}}\right)+W_{p}\left(\nu_{t_{2}}^{n}, \nu_{t_{2}}\right) \rightarrow 0 .
\end{aligned}
$$

(ii) For $s, t \geq 0$, by the triangle inequality,

$$
\begin{aligned}
W_{p}\left(\lambda, \mu_{s}\right)-s & \leq W_{p}\left(\lambda, \nu_{t}\right)+W_{p}\left(\nu_{t}, \mu_{s}\right)-s \\
& =\left[W_{p}\left(\lambda, \nu_{t}\right)-t\right]+\left[W_{p}\left(\nu_{t}, \mu_{s}\right)-s\right]+t .
\end{aligned}
$$

Letting $s \rightarrow+\infty$ and applying (i) we have

$$
\begin{aligned}
b_{\mu}(\lambda) & \leq\left[W_{p}\left(\lambda, \nu_{t}\right)-t\right]+\left[b_{\mu}\left(\nu_{t}\right)+t\right] \\
& =\left[W_{p}\left(\lambda, \nu_{t}\right)-t\right]+b_{\mu}\left(\nu_{0}\right)
\end{aligned}
$$

Let $t \rightarrow+\infty$, then the inequality follows.

For $p>1, \mathcal{X}$ is non-branching if and only if $P_{p}(\mathcal{X})$ is non-branching (see 29, Corollary 7.32]). Based on this fact, we have the following lemma.

Lemma 5.5. Assume $\mathcal{X}$ is non-branching. Let $\left(\mu_{t}\right)_{t \geq 0}$ be a unit-speed ray in $P_{p}(\mathcal{X})$. If there is another unit-speed ray $\left(\nu_{t}\right)_{t \geq 0}$ satisfying

$$
b_{\mu}\left(\nu_{t}\right)-b_{\mu}\left(\nu_{s}\right)=s-t, \text { for any } s, t \geq 0,
$$

then for any $\tau>0$, the subray $\left(\nu_{t+\tau}\right)_{t \geq 0}$ is the unique co-ray from $\nu_{\tau}$ to $\left(\mu_{t}\right)_{t \geq 0}$. 
Proof. For $\tau>0$, assume $\left(\tilde{\nu}_{t}\right)_{t \geq 0}$ is a co-ray from $\nu_{\tau}$ to $\left(\nu_{t}\right)_{t \geq 0}$. Let

$$
\nu_{t}^{\prime}=\left\{\begin{array}{lr}
\nu_{t}, & 0 \leq t \leq \tau \\
\tilde{\nu}_{t-\tau}, & t \geq \tau
\end{array}\right.
$$

We claim that $\left(\nu_{t}^{\prime}\right)_{t \geq 0}$ is a ray. Since $\left(\nu_{t}\right)_{t \geq 0}$ and $\left(\tilde{\nu}_{t}\right)_{t \geq 0}$ are unit-speed rays, it is obvious for $s, t \leq \tau$ or $s, t \geq \tau$ that

$$
W_{p}\left(\nu_{s}^{\prime}, \nu_{t}^{\prime}\right)=|s-t| .
$$

For $s<\tau<t$, by triangle inequality and the definition of rays,

$$
\begin{aligned}
W_{p}\left(\nu_{s}^{\prime}, \nu_{t}^{\prime}\right) & \leq W_{p}\left(\nu_{s}^{\prime}, \nu_{\tau}^{\prime}\right)+W_{p}\left(\nu_{\tau}^{\prime}, \nu_{t}^{\prime}\right) \\
& =W_{p}\left(\nu_{s}, \nu_{\tau}\right)+W_{p}\left(\tilde{\nu}_{0}, \tilde{\nu}_{t-\tau}\right) \\
& =(\tau-s)+(t-\tau) \\
& =t-s .
\end{aligned}
$$

On the other hand, by (5.3) and Proposition 5.4.

$$
\begin{aligned}
t-s & =(\tau-s)+(t-\tau) \\
& =\left[b_{\mu}\left(\nu_{s}\right)-b_{\mu}\left(\nu_{\tau}\right)\right]+\left[b_{\mu}\left(\tilde{\nu}_{0}\right)-b_{\mu}\left(\tilde{\nu}_{t-\tau}\right)\right] \\
& =\left[b_{\mu}\left(\nu_{s}^{\prime}\right)-b_{\mu}\left(\nu_{\tau}^{\prime}\right)\right]+\left[b_{\mu}\left(\nu_{\tau}^{\prime}\right)-b_{\mu}\left(\nu_{t}^{\prime}\right)\right] \\
& =b_{\mu}\left(\nu_{s}^{\prime}\right)-b_{\mu}\left(\nu_{t}^{\prime}\right) \\
& \leq W_{p}\left(\nu_{s}^{\prime}, \nu_{t}^{\prime}\right) .
\end{aligned}
$$

Here the last inequality follows from (5.2). This proves our claim. Since $P_{p}(\mathcal{X})$ is non-branching, the ray $\left(\nu_{t}^{\prime}\right)_{t \geq 0}$ coincides with $\left(\nu_{t}\right)_{t \geq 0}$.

Notice that (5.3) holds for every co-ray. Lemma 5.5) shows the second conclusion of Theorem 1.2

Theorem 5.6. Assume $\mathcal{X}$ is non-branching. Let $\left(\mu_{t}\right)_{t \geq 0}$ and $\left(\nu_{t}\right)_{t \geq 0}$ be two unitspeed rays in $P_{p}(\mathcal{X})$. If there exists a sequence $\left\{t_{n}\right\}$ tending to 0 such that $\left(\nu_{t+t_{n}}\right)_{t \geq 0}$ is the unique co-ray from $\nu_{t_{n}}$ to $\left(\mu_{t}\right)_{t \geq 0}$, then $\left(\nu_{t}\right)_{t \geq 0}$ is a co-ray from $\nu_{0}$ to $\left(\mu_{t}\right)_{t \geq 0}$.

Proof. Extract a decreasing subsequence of $\left\{t_{n}\right\}$, denote by $\left\{t_{n^{\prime}}\right\}$, such that $t_{n^{\prime}} \leq$ $2^{-n^{\prime}}$. Let $\tau \geq 1$ be a constant. For each $n^{\prime}$, by Lemma 5.5 $\left(\nu_{t+t_{n^{\prime}}}\right)_{t \geq 0}$ is the unique co-ray from $\nu_{t_{n^{\prime}}}$ to $\left(\mu_{t}\right)_{t \geq 0}$. Then there exist $s_{n^{\prime}}>\max \left\{\tau, n^{\prime}\right\}$ and geodesic $\left(\nu_{t}^{n^{\prime}}\right)_{0 \leq t \leq L_{n^{\prime}}} \in T\left(\nu_{t_{n^{\prime}}}, \mu_{s_{n^{\prime}}}\right)$ such that $W_{p}\left(\nu_{\tau}^{n^{\prime}}, \nu_{\tau+t_{n^{\prime}}}\right)<2^{-n^{\prime}}$, which gives

$$
W_{p}\left(\nu_{\tau}^{n^{\prime}}, \nu_{\tau}\right)<2^{-n^{\prime}+1}
$$

where $L_{n^{\prime}}=W_{p}\left(\nu_{t_{n^{\prime}}}, \mu_{s_{n^{\prime}}}\right)$.

By Theorem 4.4 there exists a subsequence $\left\{\left(\nu_{t}^{n^{\prime \prime}}\right)_{0 \leq t \leq L_{n^{\prime \prime}}}\right\}$ converging to a co-ray $\left(\lambda_{t}\right)_{t \geq 0}$ from $\nu_{0}$ to $\left(\mu_{t}\right)_{t \geq 0}$. It suffices to show that $\left(\lambda_{t}\right)_{t \geq 0}$ coincides with $\left(\nu_{t}\right)_{t \geq 0}$. We can see from (5.5) that $\lambda_{\tau}=\nu_{\tau}$. Let $\tilde{\nu}_{t}^{n^{\prime \prime}}=\nu_{t+\tau}^{n^{\prime \prime}}$, then $\left(\tilde{\nu}_{t}^{n^{\prime \prime}}\right)_{0 \leq t \leq L_{n^{\prime \prime}}-\tau}$ converges to the co-ray $\left(\lambda_{t+\tau}\right)_{t>0}$. By Lemma 5.5. $\lambda_{t}=\nu_{t}$ for every $t \geq \tau$. Thus the conclusion follows from that $P_{p}(\mathcal{X})$ is non-branching.

Combining Proposition 5.4 Lemma 5.5 and Theorem 5.6, we obtain Theorem 1.3 . 
Definition 5.7. Let $(\mathcal{Y}, d)$ be a metric space. The set

$$
\mathcal{V}(\mathcal{Y})=\left\{u: \mathcal{Y} \rightarrow \mathbb{R} \mid \text { for any } y \in \mathcal{Y}, u(y)=\min _{z \in \mathcal{Y} \backslash\{y\}}\{d(y, z)+u(z)\}\right\}
$$

is said to be the metric viscosity class of $\mathcal{Y}$.

For $u \in \mathcal{V}$ we mean that:

- for any $x$ and $y, u(x) \leq d(x, y)+u(y)$;

- for any $x$, there exists $y \neq x$ such that $u(x)=d(x, y)+u(y)$.

On a non-compact complete Riemannian manifold $(M, g), u$ is a viscosity solution to the Eikonal equation $|\nabla u|_{g}=1$ if and only if $u \in \mathcal{V}(M)$ (see for instance [15. 17]). Besides, Busemann functions on $(M, g)$ are viscosity solutions to the Eikonal equation (see e.g. [16]). Unfortunately, for $P_{p}(\mathcal{X})$ with $p \neq 2$, viscosity solutions can not be defined as usual because lack of proper differential structure. These facts motivate us to consider such a set for $P_{p}(\mathcal{X})$.

Theorem 5.8. If $\left(\mu_{t}\right)_{t \geq 0}$ is a unit-speed ray in $P_{p}(\mathcal{X})$, then $b_{\mu} \in \mathcal{V}\left(P_{p}(\mathcal{X})\right)$.

Proof. For any $\nu_{0}, \lambda \in P_{p}(\mathcal{X})$, from (5.2) we have $b_{\mu}\left(\nu_{0}\right) \leq W_{p}\left(\nu_{0}, \lambda\right)+b_{\mu}(\lambda)$. By Theorem 4.4, there exists a co-ray $\left(\nu_{t}\right)_{t \geq 0}$ from $\nu_{0}$ to $\left(\mu_{t}\right)_{t \geq 0}$. Thus by Proposition 5.4 .

$$
\begin{aligned}
b_{\mu}\left(\nu_{0}\right) & =t+b_{\mu}\left(\nu_{t}\right) \\
& =W_{p}\left(\nu_{0}, \nu_{t}\right)+b_{\mu}\left(\nu_{t}\right) \\
& =\min _{\lambda \in P_{p}(\mathcal{X}) \backslash\left\{\nu_{0}\right\}} W_{p}\left(\nu_{0}, \lambda\right)+b_{\mu}(\lambda) .
\end{aligned}
$$

\section{REFERENCES}

1. A. de Acosta, Invariance principles in probability for triangular arrays of B-valued random vectors and some applications, The Annals of Probability (1982): 346-373.

2. L. Ambrosio, J. Feng, On a class of first order HamiltonCJacobi equations in metric spaces, Journal of Differential Equations 256.7 (2014): 2194-2245.

3. L. Ambrosio, N. Gigli, and G. Savaré, Gradient flows: in metric spaces and in the space of probability measures, Springer Science \& Business Media, 2008.

4. M. Arjovsky and S. Chintala, and L. Bottou, Wasserstein gan, arXiv preprint arXiv:1701.07875 (2017)

5. V. Bangert, Geodesic rays, Busemann functions and monotone twist maps, Calculus of Variations and Partial Differential Equations 2.1 (1994): 49-63.

6. V. Bangert and P. Emmerich, Area growth and rigidity of surfaces without conjugate points, Journal of Differential Geometry 94.3 (2013): 367-385.

7. V. Bangert and E. Gutkin, Insecurity for compact surfaces of positive genus, Geometriae Dedicata 146.1 (2010): 165-191.

8. J. K. Beem, Global lorentzian geometry, Routledge, 2017.

9. J. Bertrand and B. Kloeckner, A geometric study of Wasserstein spaces: Hadamard spaces, Journal of Topology and Analysis 4.04 (2012): 515-542.

10. P. Billingsley, Convergence of probability measures, John Wiley \& Sons, 2013.

11. D. Burago, Y. Burago and S. Ivanov, A course in metric geometry, Vol. 33. American Mathematical Soc., 2001.

12. H. Busemann, The geometry of geodesics, Academic Press, New York, 1955.

13. R. Carmona and F. Delarue, Probabilistic analysis of mean-field games, SIAM Journal on Control and Optimization 51.4 (2013): 2705-2734.

14. J. Cheeger and D. Gromoll, The splitting theorem for manifolds of nonnegative Ricci curvature, Journal of Differential Geometry 6.1 (1971): 119-128. 
15. X. Cui, Viscosity solutions, ends and ideal boundaries, Illinois Journal of Mathematics 60.2 (2016): 459-480.

16. X. Cui and J. Cheng, Busemann Functions and Barrier Functions, Acta Applicandae Mathematicae (2017).

17. A, Fathi, Weak KAM theorem in Lagrangian dynamics, to appear on Cambrige studies in Advanced Mathematics, Cambrige University Press.

18. G. Galloway and A. Horta, Regularity of Lorentzian Busemann functions, Transactions of the American Mathematical Society 348.5 (1996): 2063-2084.

19. W. Gangbo, T. Nguyen and A. Tudorascu, Hamilton-Jacobi equations in the Wasserstein space, Methods and Applications of Analysis 15.2 (2008): 155-184.

20. N. Innami, Differentiability of Busemann functions and total excess, Mathematische Zeitschrift 180.3 (1982): 235-247.

21. L. Jin and X. Cui, Global viscosity solutions for eikonal equations on class A Lorentzian 2-tori, Geometriae Dedicata 193.1 (2018): 155-192.

22. S. Lisini, Characterization of absolutely continuous curves in Wasserstein spaces, Calculus of Variations and Partial Differential Equations 28.1 (2007): 85-120.

23. A. Papadopoulos, Metric spaces, convexity and nonpositive curvature, Vol. 6. European Mathematical Society, 2005.

24. P. Petersen, Riemannian geometry, Vol. 171. New York: Springer, 2006.

25. F. Santambrogio, Optimal transport for applied mathematicians, Birkäuser, NY 55 (2015): 58-63.

26. K. Shiohama, Topology of complete noncompact manifolds, Geometry of geodesics and related topics, Advanced Studies in Pure Mathematics 3 (1984): 423-450.

27. C. Sormani, Busemann Functions on Manifolds with Lower Ricci Curvature Bounds and Minimal Volume Growth, Journal of Differential Geometry 48, (1998) 557-585.

28. C. Sormani, The almost rigidity of manifolds with lower bounds on Ricci curvature and minimal volume growth, Communications in Analysis and Geometry 8.1 (2000): 159-212.

29. C. Villani, Optimal transport: old and new, Vol. 338. Springer Science \& Business Media, 2008.

30. W. Whitt, Weak convergence of probability measures on the function space $C[0, \infty)$, The Annals of Mathematical Statistics 41.3 (1970): 939-944.

Guomin Zhu, Department of Mathematics, Nanjing University, Nanjing 210093, China

E-mail address: zhu_guomin@hotmail.com

Wen-Long Li, School of Mathematics, Sun Yat-Sen University, Guangzhou, 510275, CHINA

E-mail address: liwenlongchn@gmail.com

Xiaojun Cui, Department of Mathematics, Nanjing University, Nanjing 210093, China

E-mail address: xcui@nju.edu.cn 